\title{
Combination synchronization of fractional order n-chaotic systems using active backstepping design
}

https://doi.org/10.1515/nleng-2017-0073

Received June 5, 2017; revised May 12, 2018; accepted June 26, 2018.

\begin{abstract}
In this article, a scheme using active backstepping design method is proposed to achieve combination synchronization of $n$ number of fractional order chaotic systems. In the proposed method the controllers are designed with the help of a new lemma and Lyapunov function in a systematic way. Synchronization among three/four fractional order systems have been shown as examples of synchronization of n-chaotic systems. Numerical simulation and graphical results clearly exhibit that the method of this new procedure is easy to implement and reliable for synchronization of fractional order chaotic systems.
\end{abstract}

Keywords: Backstepping method, Lyapunov stability theory, Synchronization, Fractional order chaotic systems

\section{Introduction}

The theory of fractional calculus deals with derivatives and integrals of arbitrary order and has applications in various scientific field and engineering including viscoelasticity, fluid mechanics, material science, colored noise, dielectric polarization, electromagnetic wave, bioengineering, biological model, electromechanical system, etc. [1-11]. The fractional differential equations are the generalization of classical differential equations. The advantage of fractional order system is it allows greater flexibility in the model. The fractional order differential operator is non local but integer order differential operator is a local operator in the sense that fractional order differential operator takes into account the fact that the future state

*Corresponding Author: S. Das, Department of Mathematical Sciences, Indian Institute of Technology (BHU), Varanasi-221005, India, E-mail: sdas.apm@iitbhu.ac.in

Vijay K. Yadav, Department of Mathematical Sciences, Indian Institute of Technology (BHU), Varanasi-221005, India not only depends upon the present state but also upon all of the histories of its previous states. For this realistic property, fractional calculus which was in earlier stage considered as mathematical curiosity now becomes the object of extensive development of fractional order partial differential equations for its the purpose of engineering applications.

Chaos synchronization is an interesting phenomenon of nonlinear dynamical systems and it may occur when two or more chaotic systems are coupled or one chaotic system drives the other. The synchronization of chaotic systems was first given by Pecora and Carroll [12] in 1960, and after which it has been intensively studied due to its potential applications in various fields viz., ecological system, physical system, chemical system, secure communications etc [13-22]. In recent years various types of synchronization have been investigated such as complete synchronization, anti-synchronization, lag synchronization, adaptive synchronization, projective synchronization, function projective synchronization etc [23-26] and also different schemes have been successfully applied to chaos synchronization viz., linear and nonlinear feedback control method, active control method, adaptive control method, sliding mode control method, backstepping method etc [27-34].

The method here to use is backstepping design, which has been employed by many researchers for controlling and synchronizing chaotic systems as well as hyperchaotic systems. It consists in a recursive procedure that links the choice of a Lyapunov function with the design of a controller. Backstepping design is recognised as powerful design method for chaos synchronization. The design can guarantee global stability, tracking and transient performance for a broad class of strict-feedback nonlinear systems [35-37]. To stabilize and track chaotic systems, the method had been successfully used by Mascolo and Grassi [38] in the year 1999. In 2006, the backstepping control was used by Bin et al. [39] to synchronize two coupled chaotic neurons in external electrical stimulation. Wang and $\mathrm{Ge}[40]$ proposed the Adaptive synchronization of uncertain chaotic systems via backstepping design. Back- 
stepping design was successfully applied by Tan et al. [41] during synchronization of the chaotic systems and also by $\mathrm{Yu}$ and Zhand [42] to control the uncertain behavior of chaotic systems. Recently, Park [43], Wu et al. [44] have shown that the backstepping method is very simple, reliable and powerful for controlling the chaotic behavior and synchronization of chaotic systems. But to the best of authors' knowledge the synchronization of fractional order systems using backstepping control has not yet been studied by any researcher. The theme of the present study is to investigate the synchronization procedure for a number of fractional order chaotic systems using this simple and reliable backstepping method. In our earlier article [45], active control method and backstepping approach are used to synchronize the fractional order chaotic systems. The fractional order Chen and Qi systems are taken to synchronize using both the methods. It was shown that the backstepping method takes less time to synchronize as the systems pair approaches from standard order to fractional order. This has motivated the authors to find the required time of synchronization among three/four fractional order chaotic systems.

Initially the prediction of a system had been confined through finding the analytical solution of the formal modelling of the systems via mathematical modelling with a set of parameters and initial/boundary conditions. But after the advent of modern computers and related software packages, the simulation has become a useful technique of modelling of many streams of science and engineering as well as computational sociology. Nowadays it is used in technology to optimize the performance, safety engineering, also during modelling of natural and human systems. Simulation is described as the limitation of operation of a real world system over time. Thus before performing simulation, it requires to develop a model which will represent key features of the selected physical or abstract systems. Thus simulation basically represents the operation of the system over time. During synchronization of identical or non-identical chaotic systems the simulation is used to find requirement of minimum time after which the states of slave system behave similar to the master system.

The synchronization of three chaotic dynamical systems in integer order are first studied by the Runzi et al. [46] in 2011. In 2013 Zhang et al. [47] studied the combination synchronization of different kinds of spatiotemporal coupled systems with unknown parameters. In 2016 the combination synchronization of different kinds of spatiotemporal coupled systems with unknown parameters is studied by Wang et al. [48]. It is seen from literature survey that the synchronization between three and more chaotic systems are few in numbers. Runzi et al. [46] had stated the cause of investigation between two drive systems and one response system through a physical application in secure communication as transmitted signals can be split into several parts, each part loaded in different drive systems which shows that transmitted signals have stronger anti-attack ability and anti-translated capability than that transmitted by the usual transmission model. This has motivated the authors to study the generalization of synchronization between chaotic systems, when the systems have memory effects. The main contribution of the present scientific contribution is introduction of a new type of synchronization scheme known as combination synchronization which used to synchronize a number of fractional order chaotic systems. The backstepping method is applied during synchronization of fractional order chaotic systems using Lyapunov stability theory and a new lemma for Caputo derivative. The combination synchronization of three and four fractional order chaotic systems are found through numerical simulation which is presented graphically to show the effectiveness and feasibility of the proposed scheme and method. The salient feature of the present study is the pictorial presentation of requirement of less time during synchronization of four systems compared to three systems.

The article is organized as follows: In section 2, the definition and Lemma for Caputo derivative in fractional order case are introduced. Section 3 includes the combination synchronization scheme of fractional order $\mathrm{n}$-chaotic systems are presented. In section 4, the fractional order chaotic systems are introduced. Sections 5 and 6 contain the combination synchronization of three and four chaotic systems using backstepping design respectively. Section 5.1 and 6.1 provides numerical simulation results and followed by a conclusion of the overall research work given in Section 7.

\section{Basic definitions of Fractional order derivative and Lemma}

The definitions of fractional order derivative given by B Riemann and J. Liouville and also by M. Caputo are as follows.

2.1 Definition 1. The Riemann-Liouville (R-L) fractional derivative operator of order $q>0$ of a function $f(t)$ is defined by [49]

$$
D_{\chi}^{q} f(t)=\frac{d^{n}}{d t^{n}} J_{x}^{n-q} f(t), \quad n-1<q \leq n, \quad n \in \mathrm{N},
$$


where the fractional integral operator of order $q>0$ of a function $f(t)$ is given by

$$
J_{t}^{q} f(t)=\frac{1}{\Gamma(q)} \int_{0}^{t}(t-\xi)^{q-1} f(\xi) d \xi,
$$

with $J_{t}^{0} f(t)=f(t)$..

2.2 Definition 2. The Caputo order fractional derivative of a function $f(t)$ is $[50,51]$

$$
\begin{gathered}
D_{t}^{q} f(t)=\frac{1}{\Gamma(n-q)} \int_{0}^{t}(t-\xi)^{n-q-1} f^{n}(\xi) d \xi, \\
n-1<q<n, \quad n \in \mathrm{N}, \\
D_{t}^{q} f(t)=\frac{d^{n} f(t)}{d x^{n}}, \quad q=n,
\end{gathered}
$$

where $D_{t}^{q} f(t)$ satisfies the following basic property

$$
\begin{aligned}
& \left(J_{t}^{q} D_{t}^{q}\right) f(t)=f(t)-\sum_{k=0}^{n-1} f^{(k)}(0+) \frac{t^{k}}{k !}, \\
& t \geq 0, n-1<q<n, \quad n \in \mathrm{N} \text { and } f \in C_{\mu}^{n}, \quad \mu \geq-1 .
\end{aligned}
$$

Between these two, the Caputo derivative is commonly used by the researchers. The main difference of considering the Caputo derivative is that its derivative of a constant is zero whereas in the case of finite value of lower terminal ' $a$ ' the R-L derivative of a constant is ${ }_{a} D_{t}^{q} c=\frac{c t^{-q}}{\Gamma(1-q)}$. However for the case $a \rightarrow-\infty$ this becomes zero. Thus for the steady state dynamical process fractional order derivative for both Caputo and R-L provide the same results.

Another reason of choosing Caputo derivative is that it holds for homogeneous and non-homogeneous initial conditions whereas R-L derivative require homogeneous initial condition during the solution of initial value problems. 2.3 Lemma 1: [52] Let $x(t) \in R$ be a continuous and derivable function. Then for any time instant $t \geq t_{0}$,

$$
\frac{1}{2} t_{0} D_{t}^{q} x^{2}(t) \leq x(t)_{t_{0}}^{c} D_{t}^{q} x(t), \forall q \in(0,1) .
$$

\section{The scheme of combination synchronization of fractional order $\mathbf{n}$-chaotic systems}

In this scheme, (n-1) drive systems and one response system are assumed to be in fractional order system.

The drive systems are considered as

$$
D_{t}^{q} x_{1}=f_{1}\left(x_{1}\right)
$$

$$
D_{t}^{q} x_{2}=f_{2}\left(x_{2}\right)
$$

$$
D_{t}^{q} x_{n-1}=f_{n-1}\left(x_{n-1}\right) \text {, }
$$

and the response system is taken as

$$
D_{t}^{q} x_{n}=f_{n}\left(x_{n}\right)+U\left(x_{1}, x_{2}, \ldots \ldots, x_{n}\right),
$$

where $x_{1}=\left(x_{1}^{1}, x_{2}^{1}, \ldots \ldots . ., x_{n}^{1}\right), x_{2}=\left(x_{1}^{2}, x_{2}^{2}, \ldots \ldots . ., x_{n}^{2}\right)$, $\ldots \ldots \ldots \ldots . . . ., x_{n-1}=\left(x_{1}^{n-1}, x_{2}^{n-1}, \ldots \ldots . ., x_{n}^{n-1}\right)$ and $x_{n}=\left(x_{1}^{n}, x_{2}^{n}, \ldots \ldots . ., x_{n}^{n}\right)$ with $x_{1}, x_{2}, \ldots \ldots \ldots, x_{n-1}, x_{n} \in$ $R^{n}$ are the state vectors of the n-chaotic systems. $f_{1}, f_{2}, \ldots \ldots, f_{n-1}, f_{n}: R^{n} \rightarrow R^{n}$ are the $\mathrm{n}$ continuous vector functions and $U\left(x_{1}, x_{2}, \ldots \ldots, x_{n}\right)$ : $R^{n} \times R^{n} \times \ldots \ldots \times R_{n \text { times }}^{n} R^{n}$ is a controller which will be designed latter.

Definition: The fractional order (n-1) drive systems and one response system follow combination synchronization among n-chaotic systems if there exists $n$ constant matrixes called scaling matrixes $A_{1}, A_{2}, \ldots . ., A_{n} \in R^{n}$ with $A_{n} \neq 0$ such that

$$
\lim _{n \rightarrow+\infty}\left\|A_{1} x_{1}+A_{2} x_{2}+\ldots \ldots . .-A_{n} x_{n}\right\|=0 \text {, where }\|\cdot\|
$$
represent the matrix norm.

It is noted that if $A_{1} \neq 0, A_{2}=A_{3}=\ldots \ldots \ldots=A_{n-1}=$ $0, A_{n}=I$ then this problem is reduced to the projective synchronization, where $I$ is an $n \times n$ identity matrix. If the scaling matrix $A_{1}$ is considered as a function, then synchronization problem is reduced into function projective synchronization problem. Again if $A_{1}=A_{2}=\ldots \ldots . . .=$ $A_{n-1}=0$, then the problem becomes a chaos control problem.

\section{Systems' descriptions}

\subsection{Fractional order Newton-Leipnik system}

The fractional order Newton-Leipnik system [53] was first studied in the year 2008, which is given by

$$
\begin{gathered}
\frac{d^{q} x_{1}}{d t^{q}}=-a_{1} x_{1}+x_{2}+10 x_{2} x_{3} \\
\frac{d^{q} x_{2}}{d t^{q}}=-x_{1}-0.4 x_{2}+5 x_{1} x_{3} \\
\frac{d^{q} x_{3}}{d t^{q}}=a_{2} x_{3}-5 x_{1} x_{2}, 0<q<1,
\end{gathered}
$$

where $a_{1}$ and $a_{2}$ are the variable parameters' and $a_{2} \in$ $(0,0.8)$. The system is ill-behaved when $a_{2}$ takes the values outside of this interval. If $a_{2}$ becomes close to zero, the 
system shows uninteresting dynamic and if $a_{2} \geq 0.8$, the given system becomes explosive i.e., the solution of this system diverges to infinity for any initial condition other than the critical points.

For the parameters' values $a_{1}=0.4, a_{2}=0.175$ and the initial condition $(0.19,0,-0.18)$, the NewtonLeipnik system shows chaotic behaviour at $q=0.95$ which is depicted through Fig. 1(a).

\subsection{Fractional order Liu system}

The Liu system [54] was studied in the year 2009, which was later extended to fractional order Liu system by Gejji and Bhalekar [55] in 2010 as

$$
\begin{gathered}
\frac{d^{q} y_{1}}{d t^{q}}=-b_{1} y_{1}-b_{4} y_{2}^{2} \\
\frac{d^{q} y_{2}}{d t^{q}}=b_{2} y_{2}-b_{5} y_{1} y_{3} \\
\frac{d^{q} y_{3}}{d t^{q}}=-b_{3} y_{3}+b_{6} y_{1} y_{2} .
\end{gathered}
$$

The phase portrait of the system is described through Fig.1(b), which shows that the system exhibits chaos at the lowest fractional order $q=0.92$ for the values of parameters $b_{1}=1, b_{2}=2.5, b_{3}=5, b_{4}=1, b_{5}=4, b_{6}=4$ and initial condition $(0.2,0,0.5)$. The chaos control of Liu system is given in [56]. The trajectories of the system are controlled at its all equilibrium points for order of the derivative $0<q \leq 1$.

\subsection{Fractional order Lotka-Voltra system}

The fractional order Lotka-Voltra system [57] is given as

$$
\begin{gathered}
\frac{d^{q} z_{1}}{d t^{q}}=c_{1} z_{1}-c_{2} z_{1} z_{2}+c_{5} z_{1}^{2}-c_{6} z_{3} z_{1}^{2} \\
\frac{d^{q} z_{2}}{d t^{q}}=-c_{3} z_{2}+c_{4} z_{1} z_{2} \\
\frac{d^{q} z_{3}}{d t^{q}}=-c_{7} z_{3}+c_{6} z_{3} z_{1}^{2} .
\end{gathered}
$$

The chaotic attractor of the system is described through Fig. 1(c) at $q=0.95$ for the values of the parameters $c_{1}=c_{2}=c_{3}=c_{4}=1, c_{5}=2, c_{6}=2.7, c_{7}=3$ and initial condition $(1,1.4,1)$.

\subsection{Fractional order Chen system}

The fractional order Chen system [58] is considered as

$$
\frac{d^{q} w_{1}}{d t^{q}}=d_{1}\left(w_{2}-w_{1}\right)
$$

$$
\begin{gathered}
\frac{d^{q} w_{2}}{d t^{q}}=\left(d_{3}-d_{1}\right) w_{1}-w_{1} w_{3}+d_{3} w_{2} \\
\frac{d^{q} w_{3}}{d t^{q}}=w_{1} w_{2}-d_{2} w_{3} .
\end{gathered}
$$

Fig. 1(d) shows the chaotic attractors of the system at the fractional order $q=0.95$ for the parameters' values $d_{1}=35, d_{2}=3, d_{3}=28$ and the initial condition $(1,1.4,1)$. The chaos control of Chen system is given in [58]. The system is controlled at order $q=0.9, q=0.8$ at its equilibrium points. The trajectories of the system is stable at all the equilibrium points of the system and it will also remain be controlled for order $0<q \leq 1$.

\section{Synchronization of fractional order Newton-Leipnik, Lotka-Voltra and Liu systems}

For the study of synchronization among three fractional order chaotic systems, two systems Newton-Leipnik (5) and Lotka-Voltra (7) are considered as drive system-I and drive system-II and third system Liu system is considered as response system. The response system with the control functions $u_{1}, u_{2}, u_{3}$ is defined as

$$
\begin{gathered}
\frac{d^{q} y_{1}}{d t^{q}}=-b_{1} y_{1}-b_{4} y_{2}^{2}+u_{1} \\
\frac{d^{q} y_{2}}{d t^{q}}=b_{2} y_{2}-b_{5} y_{1} y_{3}+u_{2} \\
\frac{d^{q} y_{3}}{d t^{q}}=-b_{3} y_{3}+b_{6} y_{1} y_{2}+u_{3} .
\end{gathered}
$$

Defining the error functions as $e_{i}=y_{i}-z_{i}-x_{i}, i=$ $1,2,3$, we obtain the error system as

$$
\frac{d^{q} e_{1}}{d t^{q}}=-b_{1} e_{1}-b_{4} e_{2}+\varphi_{1}+u_{1}
$$

$\frac{d^{q} e_{2}}{d t^{q}}=b_{2} e_{2}-b_{5} e_{1} e_{3}-b_{5} e_{3}\left(z_{1}+x_{1}\right)-b_{5} e_{1}\left(z_{3}+x_{3}\right)+\varphi_{2}+u_{2}$

$\frac{d^{q} e_{3}}{d t^{q}}=-b_{3} e_{3}+b_{6} e_{1} e_{2}+b_{6} e_{2}\left(z_{1}+x_{1}\right)+b_{6} e_{1}\left(z_{2}+x_{2}\right)+\varphi_{3}+u_{3}$,

where $\varphi_{1}=-b_{1}\left(z_{1}+x_{1}\right)-b_{4}\left(z_{2}+x_{2}\right)-c_{1} z_{1}+c_{2} z_{1} z_{2}-$ $c_{5} z_{1}^{2}+c_{6} z_{3} z_{1}^{2}+a_{1} x_{1}-x_{2}-10 x_{2} x_{3}$

$$
\begin{aligned}
\varphi_{2}= & -b_{5}\left(z_{1}+x_{1}\right)\left(z_{3}+x_{3}\right)+b_{2}\left(z_{2}+x_{2}\right)+c_{3} z_{2} \\
& -c_{4} z_{1} z_{2}+x_{1}+0.4 x_{2}-5 x_{1} x_{3} \\
\varphi_{3}= & b_{6}\left(z_{1}+x_{1}\right)\left(z_{2}+x_{2}\right)-b_{3}\left(z_{3}+x_{3}\right)+c_{7} z_{3} \\
& -c_{6} z_{3} z_{1}^{2}-a_{2} x_{3}+5 x_{1} x_{2} .
\end{aligned}
$$




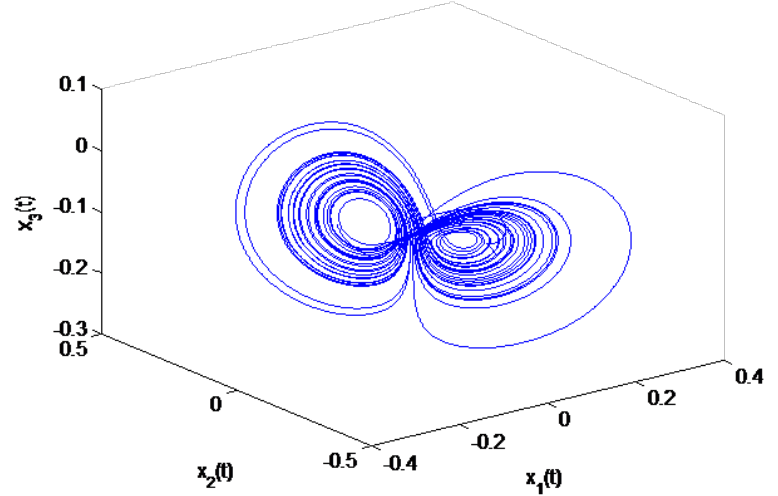

(a)

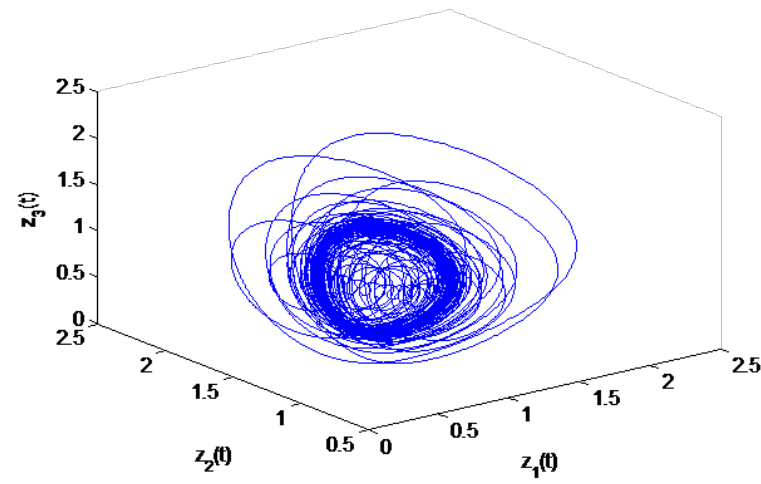

(c)

Fig. 1: Phase portraits of fractional order (a) Newton-Leipnik system, (b) Liu system, (c) Lotka-Voltra system, (d) Chen system for the order of derivative $q=0.95$.

Now the control functions would be designed using backstepping approach for combination synchronization of three fractional order chaotic systems.

Theorem 1: If the control functions are chosen as

$$
\begin{gathered}
u_{1}=-\varphi_{1}, \\
u_{2}=b_{5} v_{1}\left(z_{3}+x_{3}\right)-b_{2} v_{2}+b_{4} v_{1}-v_{2}-\varphi_{2} \\
u_{3}=-b_{6} v_{1}\left(z_{2}+x_{2}\right)+\left(b_{5}-b_{6}\right) v_{2}\left(z_{1}+x_{1}\right)+\left(b_{5}-b_{6}\right) v_{1} v_{2}-\varphi_{3},
\end{gathered}
$$

where $v_{1}=e_{1}, v_{2}=e_{2}, v_{3}=e_{3}$, then the drive system I \& II will be combination synchronized with response system.

Proof: To achieve the results, let us use the active backstepping procedure through following three steps.

Step-I: Defining $v_{1}=e_{1}$, we get

$$
\frac{d^{q} v_{1}}{d t^{q}}=\frac{d^{q} e_{1}}{d t^{q}}=-b_{1} v_{1}-b_{4} e_{2}+\varphi_{1}+u_{1},
$$

where $e_{2}=\alpha_{1}\left(v_{1}\right)$ is regarded as a virtual controller. For designing $\alpha_{1}\left(v_{1}\right)$ to stabilize $v_{1}$ - subsystem, choosing the

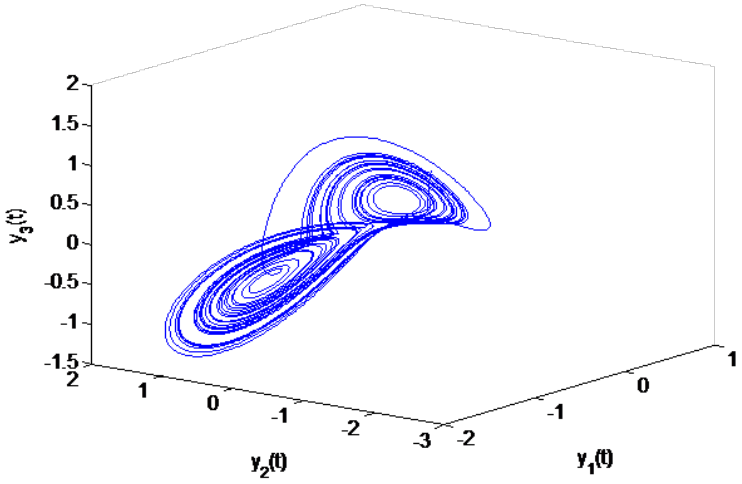

(b)

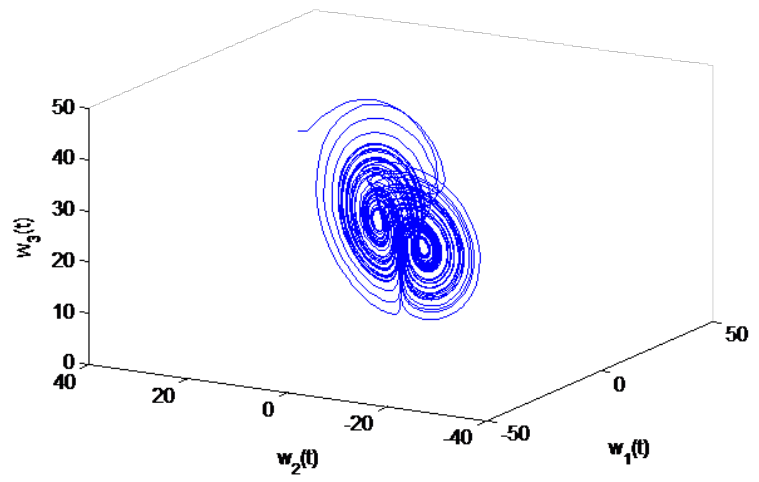

(d)

Lyapunov function $V_{1}$ as

$$
V_{1}=\frac{1}{2} v_{1}^{2} .
$$

The-th order fractional derivative of $V_{1}$ w. r. to $t$ is

$$
\begin{aligned}
\frac{d^{q} V_{1}}{d t^{q}} & =\frac{1}{2} \frac{d^{q} v_{1}^{2}}{d t^{q}} \\
& \leq v_{1} \frac{d^{q} v_{1}}{d t^{q}} \text { (using Lemma-1) } \\
& \text { i.e. } \leq v_{1}\left[-b_{1} v_{1}-b_{4} \alpha_{1}\left(v_{1}\right)+\varphi_{1}+u_{1}\right] .
\end{aligned}
$$

If we take $\alpha_{1}\left(v_{1}\right)=0$ and $u_{1}=\varphi_{1}$, then $\frac{d^{q} V_{1}}{d t^{q}} \leq-b_{1} v_{1}^{2}<$ 0 , which implies that subsystem (11) is asymptotically stable. Since virtual control function $\alpha_{1}\left(v_{1}\right)$ is an estimate function, defining the error variable $v_{2}$ between $e_{2}$ and $\alpha_{1}\left(v_{1}\right)$ as

$$
v_{2}=e_{2}-\alpha_{2}\left(v_{1}\right),
$$


we obtain the following $\left(v_{1}, v_{2}\right)$-subsystem as

$$
\begin{aligned}
\frac{d^{q} v_{1}}{d t^{q}} & =-b_{1} v_{1}-b_{4} v_{2} \\
\frac{d^{q} v_{2}}{d t^{q}} & =b_{2} v_{2}-b_{5} v_{1} e_{3}-b_{5} e_{3}\left(z_{1}+x_{1}\right) \\
& -b_{5} v_{1}\left(z_{3}+x_{3}\right)+\varphi_{2}+u_{2}
\end{aligned}
$$

where $e_{3}=\alpha_{2}\left(v_{1}, v_{2}\right)$ is regarded as an virtual controller. Step II: To stabilize $\left(v_{1}, v_{2}\right)$ - subsystem (12), choose Lyapunov function as

$$
V_{2}=V_{1}+\frac{1}{2} v_{2}^{2}=\frac{1}{2} v_{1}^{2}+\frac{1}{2} v_{2}^{2}
$$

The order fractional derivative of $V_{2}$ w. r. to $t$ is

$$
\frac{d^{q} V_{2}}{d t^{q}}=\frac{1}{2} \frac{d^{q} v_{1}^{2}}{d t^{q}}+\frac{1}{2} \frac{d^{q} v_{2}^{2}}{d t^{q}}
$$

$\leq v_{1} \frac{d^{q} v_{1}}{d t^{q}}+v_{2} \frac{d^{q} v_{2}}{d t^{q}}$, (from Lemma 1$)$

i.e. $\leq-b_{1} v_{1}^{2}-b_{4} v_{1} v_{2}+v_{2}\left[b_{2} v_{2}-b_{5} v_{1} \alpha_{2}\left(v_{1}, v_{2}\right)-\right.$ $\left.b_{5} \alpha_{2}\left(v_{1}, v_{2}\right)\left(z_{1}+x_{1}\right)-b_{5} v_{1}\left(z_{3}+x_{3}\right)+\varphi_{2}+u_{2}\right]$

If $\alpha_{2}\left(v_{1}, v_{2}\right)=0$ and $u_{2}=b_{5} v_{1}\left(z_{3}+x_{3}\right)-b_{2} v_{2}+b_{4} v_{1}-$ $v_{2}-\varphi_{2}$, then $\frac{d^{q} V_{2}}{d t^{q}} \leq-b_{1} v_{1}^{2}-v_{2}^{2}<0$, which implies that $\left(v_{1}, v_{2}\right)$-subsystem (12) is asymptotically stable.

Again defining the error variable as

$$
v_{3}=e_{3}-\alpha_{2}\left(v_{1}, v_{2}\right)
$$

the $\left(v_{1}, v_{2}, v_{3}\right)$ - subsystem becomes

$$
\begin{aligned}
\frac{d^{q} v_{1}}{d t^{q}} & =-b_{1} v_{1}-b_{4} v_{2} \\
\frac{d^{q} v_{2}}{d t^{q}} & =-v_{2}+b_{4} v_{1}-b_{5} v_{1} v_{3}-b_{5} v_{3}\left(z_{1}+x_{1}\right) \\
\frac{d^{q} v_{3}}{d t^{q}} & =-b_{3} v_{3}+b_{6} v_{1} v_{2} \\
& +b_{6} v_{2}\left(z_{1} x_{1}\right)+b_{6} v_{1}\left(z_{2}+x_{2}\right)+\varphi_{3}+u_{3}
\end{aligned}
$$

Step III: To stabilize the $\left(v_{1}, v_{2}, v_{3}\right)$ - subsystem (13), choosing the Lyapunov function $V_{3}$ as

$$
V_{3}=V_{2}+\frac{1}{2} v_{3}^{2}=\frac{1}{2} v_{1}^{2}+\frac{1}{2} v_{2}^{2}+\frac{1}{2} v_{3}^{2} .
$$

The fractional derivative of $V_{3}$ is

$$
\frac{d^{q} V_{3}}{d t^{q}}=\frac{1}{2} \frac{d^{q} v_{1}^{2}}{d t^{q}}+\frac{1}{2} \frac{d^{q} v_{2}^{2}}{d t^{q}}+\frac{1}{2} \frac{d^{q} v_{3}^{2}}{d t^{q}}
$$

$\leq v_{1} \frac{d^{q} v_{1}}{d t^{q}}+v_{2} \frac{d^{q} v_{2}}{d t^{q}}+v_{3} \frac{d^{q} v_{3}}{d t^{q}}$, (from Lemma 1)

i.e. $\leq-b_{1} v_{1}^{2}-v_{2}^{2}-b_{5} v_{1} v_{2} v_{3}-b_{5} v_{2} v_{3}\left(z_{1}+x_{1}\right)+v_{3}\left[-b_{3} v_{3}+\right.$ $\left.b_{6} v_{1} v_{2}+b_{6} v_{2}\left(z_{1}+x_{1}\right)+b_{6} v_{1}\left(z_{2}+x_{2}\right)+\varphi_{3}+u_{3}\right]$

Taking $u_{3}=-b_{6} v_{1}\left(z_{2}+x_{2}\right)+\left(b_{5}-b_{6}\right) v_{2}\left(z_{1}+x_{1}\right)+$ $\left(b_{5}-b_{6}\right) v_{1} v_{2}-\varphi_{3}$, we obtain $\frac{d^{q} V_{3}}{d t^{q}} \leq-b_{1} v_{1}^{2}-v_{2}^{2}-b_{3} v_{3}^{2}<0$. Thus the system is asymptotically stable. Thus for $v_{1}=e_{1}$, $v_{2}=e_{2}-\alpha_{1}\left(v_{1}\right)=e_{2}$ and $v_{3}=e_{3}-\alpha_{2}\left(v_{1}, v_{2}\right)=e_{3}$, the error systems $e_{i} \rightarrow 0, i=1,2,3$, which helps to obtain combination synchronization among the three considered fractional order systems.

\subsection{Numerical simulation and results}

In the numerical simulation the parameters' values of the fractional order Newton-Leipnik, Lotka-Voltra and Liu systems are taken as $a_{1}=0.4, a_{2}=0.175$, and $b_{1}=1, b_{2}=$ $2.5, b_{3}=5, b_{4}=1, b_{5}=4, b_{6}=4$ respectively. The initial conditions of two drive systems and response system are taken as $(0.19,0,-0.18)$, and $(0.2,0,0.5)$ respectively. Fig. 2 shows the synchronization among three fractional order chaotic systems are achieved through backstepping approach at $q=0.95$. Figs. 2(a), 2(b) and 2(c) depict the time response of the state trajectories $x_{i}(t)+z_{i}(t)$ and $y_{i}(t)$, where $i=1,2,3$ represent the drive systems (5), (7) and response system (9) respectively. The error states are displayed through Fig. 2(d).

\section{Synchronization of fractional order Newton-Leipnik, Liu, Lotka-Voltra systems and Chen system}

In this section to synchronize four fractional order chaotic systems, we consider fractional order Newton-Leipnik system (5), fractional order Liu system (6) and fractional order Lotka-Voltra system (7) as the drive systems I, II and III respectively. The fractional order Chen system (8) is taken as response system with control function $u_{1}^{\prime}, u_{2}^{\prime}, u_{3}^{\prime}$ as

$$
\begin{aligned}
\frac{d^{q} w_{1}}{d t^{q}} & =d_{1}\left(w_{2}-w_{1}\right)+u_{1}^{\prime} \\
\frac{d^{q} w_{2}}{d t^{q}} & =\left(d_{3}-d_{1}\right) w_{1}-w_{1} w_{3}+d_{3} w_{2}+u_{2}^{\prime} \\
\frac{d^{q} w_{3}}{d t^{q}} & =w_{1} w_{2}-d_{2} w_{3}+u_{3}^{\prime} .
\end{aligned}
$$

Defining error functions as $e_{i}=w_{i}-z_{i}-y_{i}-x_{i}, i=1,2,3$, we obtain the error system as

$$
\begin{aligned}
\frac{d^{q} e_{1}}{d t^{q}} & =d_{1}\left(e_{2}-e_{1}\right)+\psi_{1}+u_{1}^{\prime} \\
\frac{d^{q} e_{2}}{d t^{q}} & =\left(d_{3}-d_{1}\right) e_{1}-e_{1} e_{3}-e_{3}\left(z_{1}+y_{1}+x_{1}\right) \\
& -e_{1}\left(z_{3}+y_{3}+x_{3}\right)+d_{3} e_{2}+\psi_{2}+u_{2}^{\prime} \\
\frac{d^{q} e_{3}}{d t^{q}} & =e_{1} e_{2}+e_{2}\left(z_{1}+y_{1}+x_{1}\right)+e_{1}\left(z_{2}+y_{2}+x_{2}\right) \\
& -d_{2} e_{3}+\psi_{3}+u_{3}^{\prime},
\end{aligned}
$$

where

$$
\begin{aligned}
\psi_{1}= & d_{1} z_{2}+\left(d_{1} b_{4} y_{2}\right) y_{2}+\left(d_{1}-1\right) x_{2}-\left(d_{1}+c_{1}\right) z_{1} \\
& -\left(d_{1}-b_{1}\right) y_{1}-\left(d_{1}-a_{1}\right) x_{1}+c_{2} z_{1} z_{2}-c_{5} z_{1}^{2}+c_{6} z_{3} z_{1}^{2} \\
& -10 x_{2} x_{3}
\end{aligned}
$$



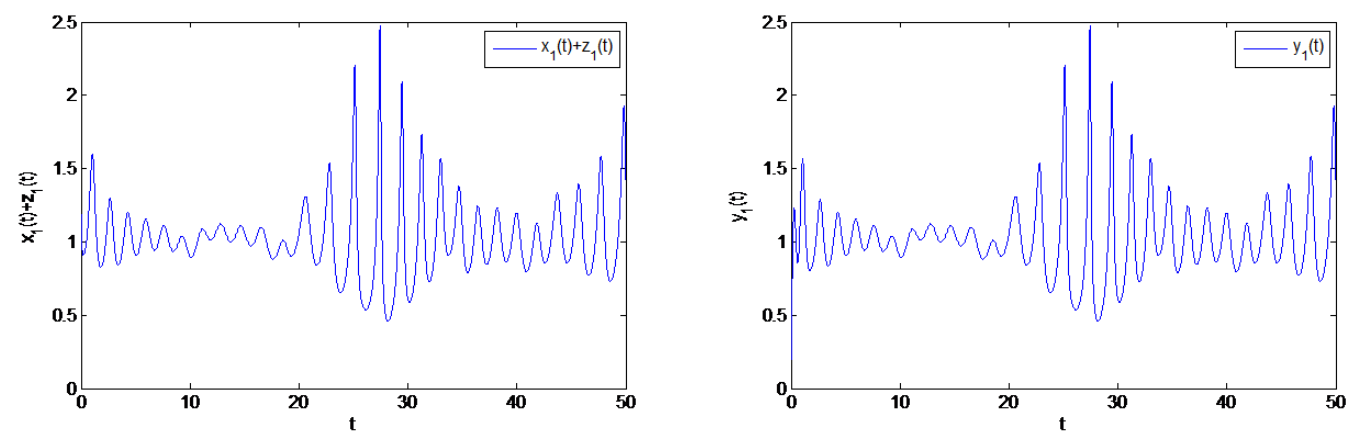

(a)
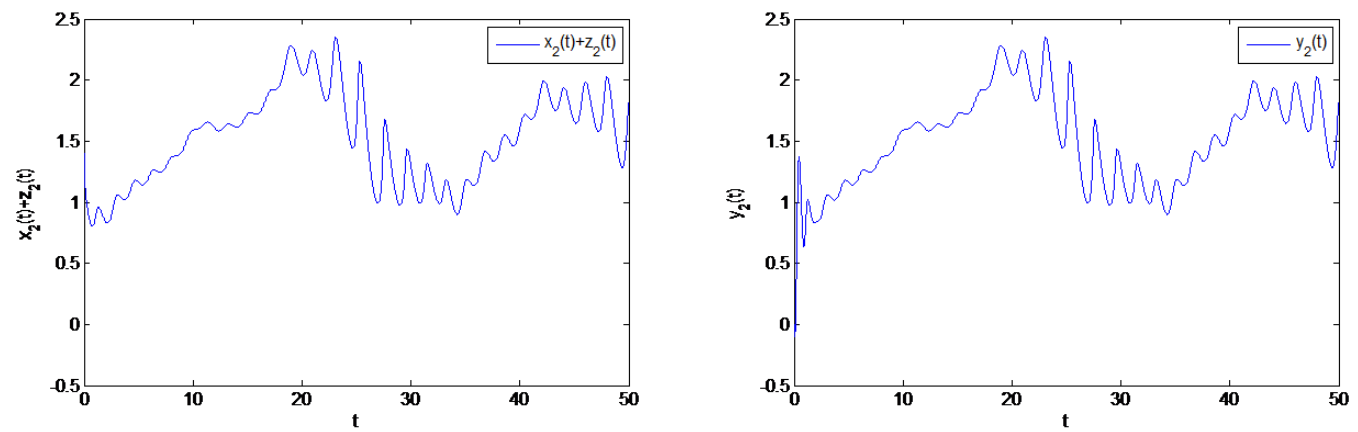

(b)
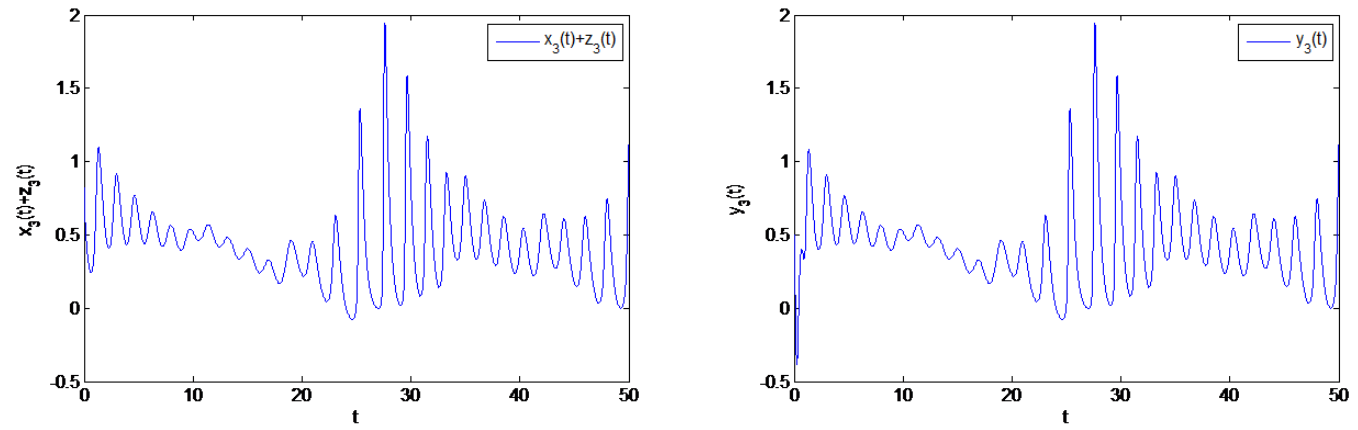

(c)

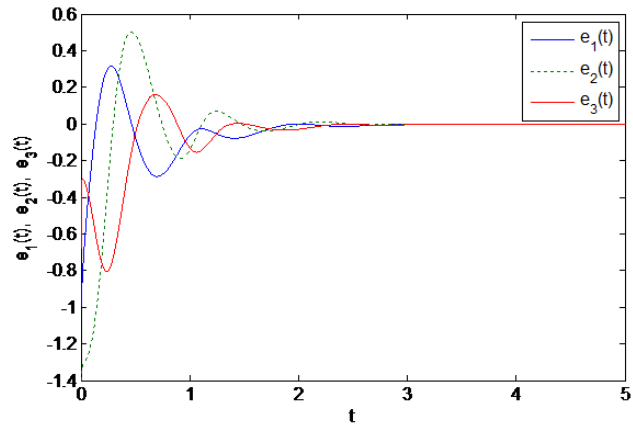

(d)

Fig. 2: Combination synchronization among three fractional order chaotic systems (5), (6) and (7) for fractional order $q=0.95$ : (a) between $x_{1}(t)+z_{1}(t)$ and $y_{1}(t)$, (b) between $x_{2}(t)+z_{2}(t)$ and $y_{2}(t)$, (c) between $x_{3}(t)+z_{3}(t)$ and $y_{3}(t)$, (d) the evaluation of error function $e_{1}(t)$, $e_{2}(t)$ sand $e_{3}(t)$. 


$$
\begin{aligned}
\psi_{2}= & \left(d_{3}-d_{1}\right)\left(z_{1}+y_{1}+x_{1}\right)-\left(z_{1}+y_{1}+x_{1}\right)\left(z_{3}+y_{3}+x_{3}\right) \\
& +d_{3}\left(z_{2}+y_{2}+x_{2}\right)+c_{3} z_{2}-c_{4} z_{1} z_{2}-b_{2} y_{2}+b_{5} y_{1} y_{3} \\
& +x_{1}+0.4 x_{2}-5 x_{1} x_{3}
\end{aligned}
$$

$$
\begin{aligned}
\psi_{3}= & \left(z_{1} y_{1} x_{1}\right)\left(z_{2}+y_{2}+x_{2}\right)-d_{2}\left(z_{3}+y_{3}+x_{3}\right)+c_{7} z_{3}-c_{6} z_{3} z_{1}^{2} \\
& +b_{3} y_{3}-b_{6} y_{1} y_{2}-a_{2} x_{3}+5 x_{1} x_{2}
\end{aligned}
$$

Next the control functions $u_{1}^{\prime}, u_{2}^{\prime}$ and $u_{3}^{\prime}$ would be designed using backstepping approach for combination synchronization of four fractional order chaotic systems.

Theorem: $\mathbf{2}$ If the control functions are chosen as

$$
\begin{gathered}
u_{1}^{\prime}=-\psi_{1}, \\
u_{2}^{\prime}=-d_{3} v_{2}+v_{1}\left(z_{3}+y_{3}+x_{3}\right)-d_{2} v_{1}-v_{2}-\psi_{2}, \\
u_{3}^{\prime}=-v_{1}\left(z_{2}+y_{2}+x_{2}\right)-\psi_{3}
\end{gathered}
$$

where $v_{1}=e_{1}, v_{2}=e_{2}, v_{3}=e_{3}$, then the drive systems (5), (6) and (7) will be combination synchronized with response system (14).

Proof: For synchronization, backstepping procedure is used through following steps.

Step-I: Considering $v_{1}=e_{1}$,

$$
\frac{d^{q} v_{1}}{d t^{q}}=\frac{d^{q} e_{1}}{d t^{q}}=d_{1}\left(e_{2}-e_{1}\right)+\psi_{1}+u_{1}^{\prime}
$$

where $e_{2}=\alpha_{1}\left(v_{1}\right)$ is regarded as a virtual controller. To stabilize $v_{1}$-subsystem, let us define the Lyapunov function $V_{1}$ as

$$
V_{1}=\frac{1}{2} v_{1}^{2},
$$

whose fractional derivative is

$$
\begin{aligned}
& \frac{d^{q} V_{1}}{d t^{q}}=\frac{1}{2} \frac{d^{q} v_{1}^{2}}{d t^{q}} \leq v_{1} \frac{d^{q} v_{1}}{d t^{q}} \\
& \text { i.e., } \leq v_{1}\left[d_{1}\left(\alpha_{1}\left(v_{1}\right)-v_{1}\right)+\psi_{1}+u_{1}^{\prime}\right]
\end{aligned}
$$

Taking $\alpha_{1}\left(v_{1}\right)=0$ and $u_{1}^{\prime}=-\psi_{1}$, we get $\frac{d^{q} V_{1}}{d t^{q}} \leq-d_{1} v_{1}^{2}<$ 0 , which implies that $v_{1}$-subsystem (16) is asymptotically stable. For the virtual control function $\alpha_{1}\left(v_{1}\right)$, a variable $v_{2}$ between $e_{2}$ and $\alpha_{1}\left(v_{1}\right)$ is defined as

$$
v_{2}=e_{2}-\alpha_{1}\left(v_{1}\right)
$$

Then $\left(v_{1}, v_{2}\right)$-subsystem is obtained as

$$
\begin{aligned}
\frac{d^{q} v_{1}}{d t^{q}} & =d_{1}\left(v_{2}-v_{1}\right) \\
\frac{d^{q} v_{2}}{d t^{q}} & =\left(d_{3}-d_{1}\right) v_{1}-v_{1} e_{3}-e_{3}\left(z_{1}+y_{1}+x_{1}\right) \\
& -v_{1}\left(z_{3}+y_{3}+x_{3}\right)+d_{3} v_{2}+\psi_{2} u_{2}^{\prime}
\end{aligned}
$$

Let us consider $v_{3}=\alpha_{2}\left(v_{1}, v_{2}\right)$ is a virtual controller.
Step II: In this step to stabilize $\left(v_{1}, v_{2}\right)$-subsystem (17), let us define the Lyapunov function $V_{2}$ as

$$
V_{2}=V_{1}+\frac{1}{2} v_{2}^{2}=\frac{1}{2} v_{1}^{2}+\frac{1}{2} v_{2}^{2} \text {. }
$$

Now

$$
\begin{aligned}
\frac{d^{q} V_{3}}{d t^{q}} & =\frac{1}{2} \frac{d^{q} v_{1}^{2}}{d t^{q}}+\frac{1}{2} \frac{d^{q} v_{2}^{2}}{d t^{q}} \\
& \leq v_{1} \frac{d^{q} v_{1}}{d t^{q}}+v_{2} \frac{d^{q} v_{2}}{d t^{q}} \\
& \text { i.e., } \leq d_{1} v_{1} v_{2}-d_{1} v_{1}^{2}+v_{2}\left[\left(d_{3}-d_{1}\right) v_{1}-v_{1} \alpha_{2}\left(v_{1}, v_{2}\right)\right. \\
& -\alpha_{2}\left(v_{1}, v_{2}\right)\left(z_{1}+y_{1}+x_{1}\right)-v_{1}\left(z_{3}+y_{3}+x_{3}\right) \\
& \left.+d_{3} v_{2}+\psi_{2}+u_{2}^{\prime}\right]
\end{aligned}
$$

Taking $\alpha_{2}\left(v_{1}, v_{2}\right)=0$ and $u_{2}^{\prime}=-d_{3} v_{2}+v_{1}\left(z_{3}+y_{3}+x_{3}\right)-$ $d_{2} v_{1}-v_{2}-\psi_{2}$, we get $\frac{d^{q} V_{2}}{d t^{q}} \leq-d_{1} v_{1}^{2}-v_{2}^{2}<0$, which makes subsystem (17) asymptotically stable.

Considering $v_{3}=e_{3}-\alpha_{2}\left(v_{1}, v_{2}\right)$, the $\left(v_{1}, v_{2}, v_{3}\right)$ - subsystem is obtained as

$$
\begin{aligned}
\frac{d^{q} v_{1}}{d t^{q}} & =d_{1}\left(v_{2}-v_{1}\right) \\
\frac{d^{q} v_{2}}{d t^{q}} & =-v_{2}-d_{1} v_{1}-v_{1} v_{3}-v_{3}\left(z_{1}+y_{1}+x_{1}\right) \\
\frac{d^{q} v_{3}}{d t^{q}} & =v_{1} v_{2}+v_{2}\left(z_{1}+y_{1} x_{1}\right)+v_{1}\left(z_{2}+y_{2}+x_{2}\right) \\
& -d_{2} v_{3}+\psi_{3}+u_{3}^{\prime} .
\end{aligned}
$$

Step III: In order to stabilize $\left(v_{1}, v_{2}, v_{3}\right)$ - subsystem (18), choosing the Lyapunov function as

$$
V_{3}=V_{2}+\frac{1}{2} v_{3}^{2}=\frac{1}{2} v_{1}^{2}+\frac{1}{2} v_{2}^{2}+\frac{1}{2} v_{3}^{2},
$$

we get

$$
\begin{aligned}
& \frac{d^{q} v_{3}}{d t^{q}}=\frac{1}{2} \frac{d^{q} v_{1}^{2}}{d t^{q}}+\frac{1}{2} \frac{d^{q} v_{2}^{2}}{d t^{q}}+\frac{1}{2} \frac{d^{q} v_{3}^{2}}{d t^{q}} \\
& \leq v_{1} \frac{d^{q} v_{1}}{d t^{q}}+v_{2} \frac{d^{q} v_{2}}{d t^{q}}+v_{3} \frac{d^{q} v_{3}}{d t^{q}}, \\
& \text { i.e., } \leq v_{1}\left[d_{1}\left(v_{2}-v_{1}\right)\right]+v_{2}\left[-v_{2}-d_{1} v_{1}-v_{1} v_{3}\right. \\
& \left.-v_{3}\left(z_{1}+y_{1}+x_{1}\right)\right]+v_{3}\left[v_{1} v_{2}+v_{2}\left(z_{1}+y_{1}+x_{1}\right)\right. \\
& \left.+v_{1}\left(z_{2}+y_{2}+x_{2}\right)-d_{2} v_{3}+\psi_{3}+u_{3}^{\prime}\right] \\
& =-d_{1} v_{1}^{2}-v_{2}^{2}+v_{3}\left[v_{1}\left(z_{2}+y_{2}+x_{2}\right)-d_{2} v_{3}+\psi_{3}+u_{3}^{\prime}\right]
\end{aligned}
$$

If $u_{3}^{\prime}=-v_{1}\left(z_{2}+y_{2}+x_{2}\right)-\psi_{3}, \frac{d^{q} V_{3}}{d t^{q}} \leq-d_{1} v_{1}^{2}-v_{2}^{2}-d_{2} v_{3}^{2}<0$, negative definite. In view of $v_{1}=e_{1}, v_{2}=e_{2}-\alpha_{1}\left(v_{1}\right)=$ $e_{2}, v_{3}=e_{2}-\alpha_{2}\left(v_{1}, v_{2}\right)=e_{3}$, the errors states $e_{i} \rightarrow$ $0, i=1,2,3$ will converge to zero after a finite period of time, and thus the combination synchronization among four fractional order chaotic systems will be achieved.

\subsection{Numerical simulation and results}

During synchronization the earlier values of the parameters and initial conditions for drive systems I, II, III and re- 

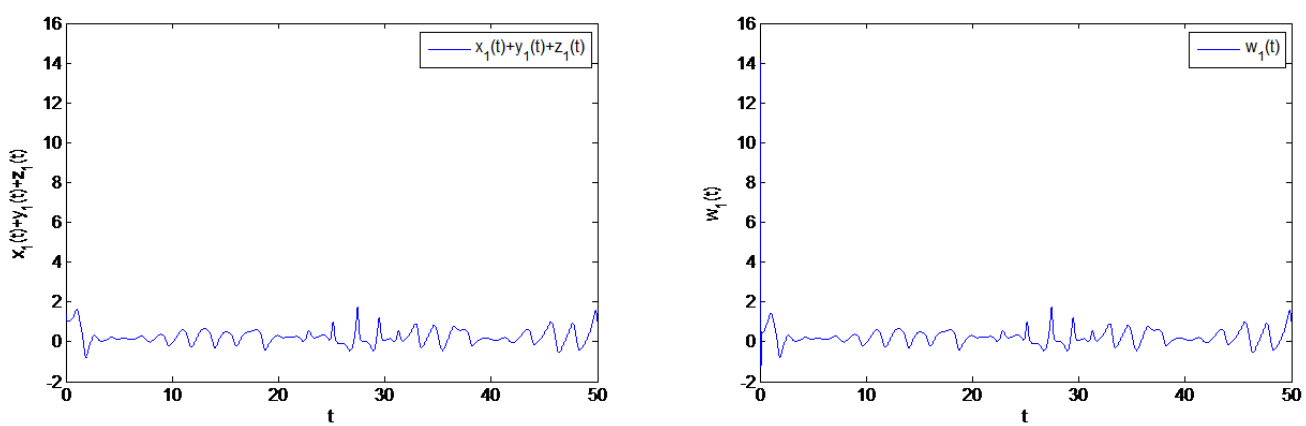

(a)
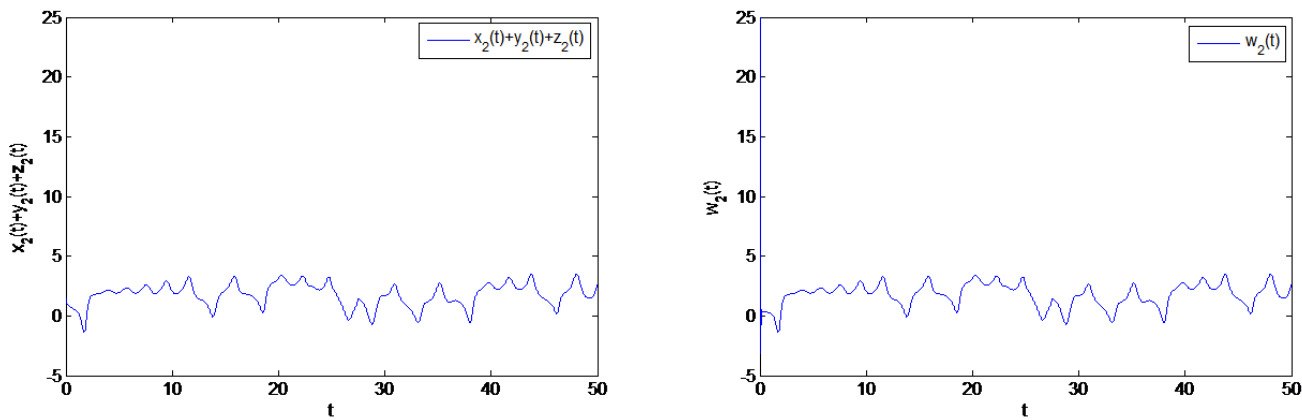

(b)
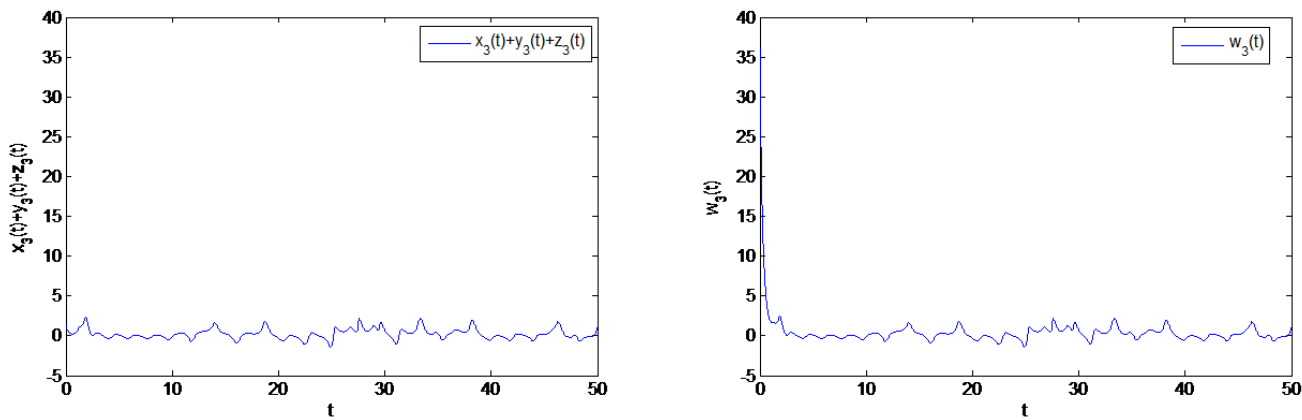

(c)

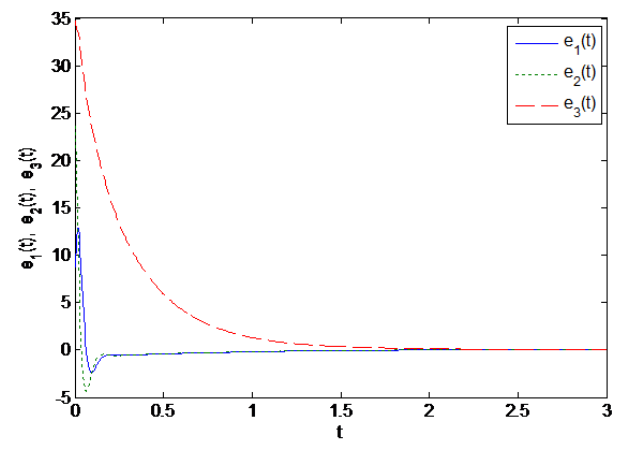

(d)

Fig. 3: Combination synchronization among four fractional order chaotic systems (5), (6), (7) and (8) for fractional order $q=0.95$ : (a) between $x_{1}(t)+y_{1}(t)+z_{1}(t)$ and $w_{1}(t)$, (b) between $x_{2}(t)+y_{2}(t)+z_{2}(t)$ and $w_{2}(t)$, (c) between $x_{3}(t)+y_{3}(t)+z_{3}(t)$ and $w_{3}(t)$, (d) the evaluation of error functions $e_{1}(t), e_{2}(t)$ and $e_{3}(t)$. 


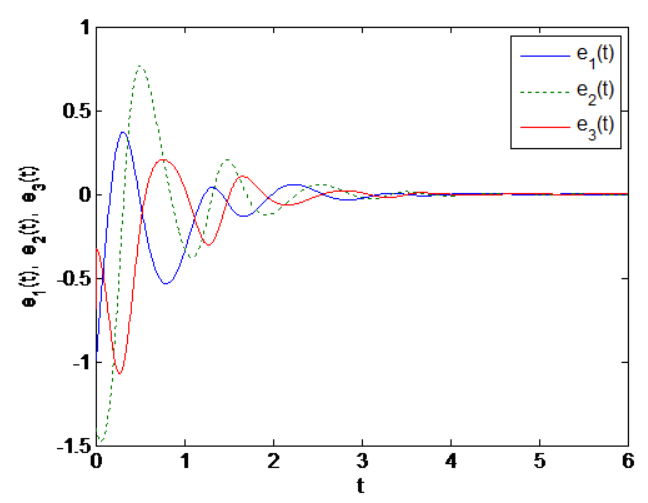

(a)

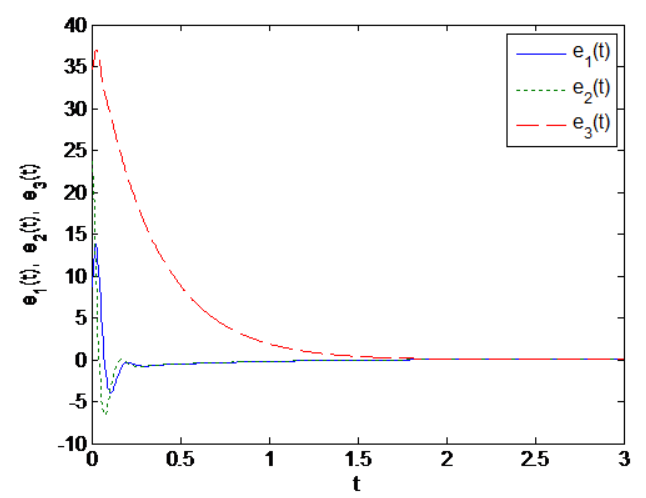

(b)

Fig. 4: The evaluation of error functions $e_{1}(t), e_{2}(t)$ and $e_{3}(t)$ at $q=1$ : (a) for three systems; (b) for four systems.

sponse system are considered. The time step size is taken as 0.005 . The synchronization among four fractional order chaotic systems are achieved through Fig. 3 using the same method at $q=0.95$. Figs. 3(a), 3(b) and 3(c) show the time response of the states $x_{i}(t)+y_{i}(t)+{ }_{i}(t)$ and $w_{i}(t)$, where $i=1,2,3$ represent the drive systems (5), (6), (7) and the response system (8). The error states for this case are described through Fig. 3(d). It is noticed that it takes less time for synchronization among four systems (Fig. 3(d)) compared to that of three systems (Fig. 2(d)) for the considered systems in both fractional order as well as integer order case (Fig. 4).

\section{Conclusion}

In the present study, the combination synchronization among a number of fractional order drive and response systems is successfully demonstrated using backstepping method. For validation, the combination synchronization of three and four systems are considered separately taking two systems and three systems as drive system respectively, while one system as response system, which clearly exhibit that the applied method is effective and convenient to achieve global synchronization of a number of non-identical fractional order chaotic systems. The exhibition of requirement of less time during synchronization of four systems compared to three systems is one of the major contributions of the present study. It is worth mentioning that this scientific contribution of combination synchronization among the fractional order chaotic systems will be significant to the research community involved in the area of modelling of fractional order dynamical systems.

Acknowledgement: The authors are extending their heartfelt thanks to the revered reviewers for their valuable comments to upgrade the present manuscript.

\section{References}

[1] Bagley, R.L., Calico, R.A., Fractional order state equations for the control of viscoelastically damped structures. J. Guid. Control Dyn., 1991, 14, 304-311.

[2] Koeller, R.C., Application of fractional calculus to the theory of viscoelasticity. J. Appl. Mech., 1984, 51, 199.

[3] Kulish, V.V., Lage, J.L., Application of fractional calculus to fluid mechanics. J. Fluids Eng., 2002, 124, 803-806.

[4] Das, S., Tripathi, D., Pandey, S.K., Peristaltic flow of viscoelastic fluid with fractional maxwell model through a channel. Appl. Math. Comput., 2010, 215, 3645-3654.

[5] Carpinteri, A., Cornetti, P., Kolwankar, K.M., Calculation of the tensile and flexural strength of disordered materials using fractional calculus. Chaos Solitons \& Fractals, 2004, 21, 623632.

[6] Sun, H.H., Abdelwahed, A.A., Onaral, B., Linear approximation for transfer function with a pole of fractional order. IEEE Trans. Automat. Control, 1984, 29, 441-444.

[7] Heaviside, O., Electromagnetic Theory. New York, Chelsea, 1971.

[8] Magin, R.L., Fractional calculus in bioengineering, Part 3. Crit. Rev. Biomed. Eng., 2004, 32, 195-377.

[9] Magin, R.L., Fractional calculus models of complex dynamics in biological tissues. Comput. Math. Appl., 2010, 59, 1585-1593.

[10] Gokdogan, A., Merdan, M., Yildirim, A., A multistage differential transformation method for approximate solution of Hantavirus infection model. Commun. Nonlinear Sci. Numer. Simul., 2012, 17, 1-8.

[11] Sabatier, J., Poullain, S., Latteux, P., Thomas J.L., Oustaloup, A., Robust speed control of a low damped electromechanical system based on CRONE control: application to a four mass experimental test bench. Nonlinear Dyn., 2004, 38, 383-400.

[12] Pecora, L.M., Carroll, T.L., Synchronization in chaotic systems, Phys. Rev. Lett., 1990, 64, 821-825. 
[13] Blasius, B., Huppert A., Stone, L., Complex dynamics and phase synchronization in spatially extended ecological system. Nature, 1999, 399, 354-359.

[14] Lakshmanan M., Murali, K., Chaos in nonlinear oscillators: controlling and synchronization. Singapore: World Scientific, 1996.

[15] Han, S.K., Kerrer C., Kuramoto, Y., D-phasing and bursting in coupled neural oscillators. Phys Rev Lett., 1995, 75, 3190-3,

[16] Cuomo, K.M., Oppenheim, A.V., Circuit implementation of synchronized chaos with application to communication. Phys Rev Lett., 1993, 71, 65-8.

[17] Murali, K., Lakshmanan, M.P., Secure communication using a compound signal using sampled-data feedback. Appl Math Mech., 2003, 11, 1309-15.

[18] Agrawal, S.K., Srivastava, M., Das, S., Synchronization between fractional-order Ravinovich-Fabrikant and LotkaVolterra systems. Nonlinear Dyn. 2012, 69, 2277-2288.

[19] Chang, C.M., Chen, H.K., Chaos and hybrid projective synchronization of commensurate and incommensurate fractionalorder Chen-Lee systems. Nonlinear Dyn. 2010, 62, 851-858.

[20] Cai, G., Hu, P., Li, Y., Modified function lag projective synchronization of a financial hyperchaotic system. Nonlinear Dyn. 2012, 69, 1457-1464.

[21] Srivastava, M., Ansari, S.P., Agrawal, S.K., Das, S., Leung, A.Y.T., Anti-synchronization between identical and nonidentical fractional-order chaotic systems using active control method. Nonlinear Dyn. 2014, 76, 905-914.

[22] Luo C., Wang, X.Y., Chaos in the fractional-order complex Lorenz system and its synchronization, Nonlinear Dyn. 2013, 71, 241-257.

[23] Wang, X.Y., Song, J.M., Synchronization of the fractional order hyperchaos Lorenz systems with activation feedback control, Commun Nonlinear Sci Numer Simulat. 2009, 14, 3351-3357.

[24] Wang, X.Y., He, Y.J., Projective synchronization of fractional order chaotic system based on linear separation, Physics Letters A, 2008, 372, 435-441.

[25] Das, S., Yadav, V.K., Stability Analysis, Chaos Control of Fractional Order Vallis and El-Nino Systems and Their Synchronization, IEEE/CAA Journal of Automatica Sinica, 2017, 4 (1), 114-124.

[26] Wang, X.Y., Zhang, X., Ma, C., Modified projective synchronization of fractional-order chaotic systems via active sliding mode control, Nonlinear Dyn. 2012, 69, 511-517.

[27] Chen S.H., Lu, J., Parameters identification and synchronization of chaotic systems based upon adaptive control. Phys. Lett. A, 2002, 299, 353-8.

[28] Huang, L., Feng, R., Wang, M., Synchronization of chaotic systems via nonlinear control. Phys. Lett. A, 2004, 320, 271-5.

[29] Agrawal, S.K., Srivastava, M., Das, S., Synchronization of fractional order chaotic systems using active control method. Chaos Solitons \& Fractals, doi:10.1016/j.chaos.2012.02.004, 2012.

[30] Chen, S.H., Lu, J.: Synchronization of an uncertain unified chaotic system via adaptive control. Chaos Solitons \& Fractals, 2002, 14, 643-7.

[31] Razminia, A., Baleanu, D., Complete synchronization of commensurate fractional order chaotic systems using sliding mode control. Mechatronics, 2013, 23, 873-879.

[32] Park, J.H., Synchronization of Genesio chaotic system via backstepping approach. Chaos \& Solitons and Fractals, 2006, 27,
1369-1375.

[33] Lin, D., Wang, X., Nian, F., Zhang Y., Dynamic fuzzy neural networks modeling and adaptive backstepping tracking control of uncertain chaotic systems, Neurocomputing, 2010, 73, 28732881.

[34] Tang, Q., Wang, X., Backstepping generalized synchronization for neural network with delays based on tracing control method, Neural Comput \& Applic, 2014, 24, 775-778.

[35] Zhang, H., Ma, X., Li, M., Zou, J., Controlling and tracking hyperchaotic Rossler system via active backstepping design. Chaos Solitons and Fractals, 2005, 26, 353-361.

[36] Kokotovic, P.V., The joy of feedback: nonlinear and adaptive. IEEE Control Syst Mag, 1992, 6, 7-17.

[37] Krstic, M., Kanellakopoulus, I., Kokotovic, P., Nonlinear and adaptive control design John Wiley, New York, 1995.

[38] Mascolo, S., Grassi, G., Controlling chaotic dynamics using backstepping design with application to the Lorenz system and Chua's circuit. Int J Bifur Chaos, 1999, 9, 1425-1434.

[39] Bin, D., Jiang, W., Xiang yang, F., Synchronizing two coupled chaotic neurons in external electrical stimulation using backstepping control, Chaos Solitons \& Fractals, 2006, 29, 182-189.

[40] Wang, C., Ge, S.S., Adaptive synchronization of uncertain chaotic systems via backstepping design, Chaos Solitons \& Fractals, 2001, 12, 1199-206.

[41] Tan, X.H., Zhang, J.Y., Yang, Y.R., Synchronization chaotic systems using backstepping design, Chaos Solitons \& Fractals, 2003, 16, 37-45.

[42] Yu, Y.G., Zhang, S.C., Controlling uncertain system using backstepping design, Chaos Solitons \& Fractals, 2003, 15, 897902.

[43] Park, J.H., Synchronization of Genesio chaotic system via backstepping approach, Chaos Solitons \& Fractals, 2006, 27, 1369-1375.

[44] Wu, Y., Zhou, X., Chen, J., Hui, B., Chaos synchronization of a new 3D chaotic system, Chaos Solitons \& Fractals, 2009, 42, 1812-1819.

[45] Singh, A.K. Yadav, V.K., Das, S., Comparative study of synchronization methods of fractional order chaotic systems, Nonlinear Engineering, 2016, 5(3), 185-192.

[46] Runzi, L., Yinglan, W., Shucheng, D., Combination synchronization of three classic chaotic systems using active backstepping design. Chaos, 2011, 21, 043114.

[47] Zhang H., Wang X., Lin X., Combination synchronization of different kinds of spatiotemporal coupled systems with unknown parameters, IET Control Theory and Applications, 2013, doi: 10.1049/iet-cta.2013.0533

[48] Wang, S., Wang, X., Wang, X., Zhou, Y., Adaptive generalized combination complex synchronization of uncertain real and complex nonlinear systems, AIP ADVANCES, 2016, 6, 045011.

[49] Podlubny, I. Fractional Differential Equations, Academic Press, San Diego. CA, 1999.

[50] Gorenflo, R., Mainradi, F., Essentials of fractional calculus. Preprint submitted to Maphysto centre, Preliminary version 2000.

[51] Oldham, K., Spanier, J., The fractional calculus, Academic Press. New York- London 1974.

[52] Norelys, A.C., Manuel, A.D.M., Gallegos, J.A., Lyapunov functions for fractional order systems. Commun Nonlinear Sci. Numer. Simulat. 2014, 19, 2951-2957. 
[53] Sheu, L.J., Chen, H.K., Chen, J.H., Tam, L.M., Chen, W.C., Lin, K.T., Kang, Y., Chaos in the Newton-Leipnik system with fractional order. Chaos, Solitons and Fractals, 2008, 36, 98-103.

[54] Liu, C., Liu, L., Liu, T., A novel three-dimensional autonomous chaos system. Chaos, Solitons and Fractals, 2009, 39, 19501958.

[55] Gejji, V.D., Bhalekar, S., Chaos in fractional ordered Liu system. Computers and Mathematics with Applications, 2010, 59, 1117-1127.
[56] Wang, X.Y., Wang, M.J., Dynamic analysis of the fractionalorder Liu system and its synchronization, CHAOS, 2007, 17, 033106.

[57] Petras, I., Fractional order nonlinear systems, modelling, analysis and simulation. Beijing, Berlin, Heidelberg: Higher education press, Springer-Verlag, 2011.

[58] Li, C., Chen, G., Chaos in the fractional order Chen system and its control. Chaos, Solitons and Fractals, 2004, 22, 549-554. 